\title{
EFFECTS OF NEONATAL CAPSAICIN TREATMENT ON NOCICEPTIVE THRESHOLDS IN THE RAT ${ }^{1}$
}

\author{
J. I. NAGY ${ }^{2}$ AND D. VAN DER KOOY \\ Medical Research Council Neurochemical Pharmacology Unit, Medical Research Council Centre, Medical School, Hills \\ Road, Cambridge, United Kingdom CB2 $2 Q H$
}

Received July 8, 1982; Revised October 18, 1982; Accepted December 28, 1982

\begin{abstract}
The responsiveness to noxious thermal, mechanical, and chemical stimuli was examined in adult rats that had been treated neonatally with capsaicin. A range of capsaicin doses was employed to determine whether the neurotoxicity of capsaicin to primary afferent fibers would be manifested behaviorally in a selective effect on nociceptive thresholds to specific stimuli. Animals were given 5 to $100 \mathrm{mg} / \mathrm{kg}$ of capsaicin at 2 days of age and were examined 2 to 4 months later using the tail flick and hot plate tests to determine thermal thresholds, the paw pressure test to determine mechanical thresholds, and the formalin test to determine chemical thresholds. Significant impairments of treated animals' responses to all three types of stimuli were found at high doses of capsaicin and at doses which seem to lead to the depletion of only unmyelinated primary afferent fibers. Slightly higher doses of capsaicin were required to increase thermal nociceptive thresholds in the tail flick test as compared with the other tests, and mechanical nociception seemed to be the most sensitive to the effects of capsaicin. At any particular dose of capsaicin, considerable variability was found in the responsiveness of animals to noxious stimuli. This may partly explain the inconsistencies in studies of nociceptive thresholds in capsaicin-treated animals. The results also demonstrate the difficulty of correlating the degree of analgesia exhibited by these animals with the extent of loss of primary afferent fibers or with the depletion of afferent putative peptide transmitters.
\end{abstract}

With the demonstration that capsaicin administered to neonatal animals causes the degeneration of primary afferent neurones and leads to a permanent loss of these through the animal's lifetime (Jancsó et al., 1977) came reports of the behavioral deficits exhibited by animals given capsaicin (Holzer et al., 1979; Nagy et al., 1980). The impetus for examining nociceptive thresholds was undoubtedly provided by the observations that primarily unmyelinated fibers were affected by capsaicin treatment and that these fibers are involved in the transmission of noxious stimuli from the periphery to the CNS (Bessou and Perl, 1969; Burgess and Perl, 1973; Hallin and Torebjork, 1973; Torebjork and Hallin, 1974). In addition, there was circumstantial evidence that unmyelinated fibers

\footnotetext{
${ }^{1}$ We would like to thank P. Barenz for typing the manuscript, T. Johnson in the Medical Research Council Statistics Unit for help in the computer analysis of the results, and A. Hayes for the loan of apparatus to determine nociceptive mechanical thresholds. J. I. N. is a Fellow of the Medical Research Council of Canada and D. van der K. is a Medical Research Council Scholar.

${ }^{2}$ To whom correspondence should be addressed at his present address: Department of Physiology, Faculty of Medicine, University of Manitoba, 770 Bannatyne Avenue, Winnipeg, Manitoba, Canada R3E 0W3.
}

contain the peptide substance $P$, which itself has been implicated as a transmitter of noxious stimuli in primary afferents (Henry, 1976). It has been hoped that neonatal capsaicin treatment would offer immediate insight into the function of these fibers and of their constituent putative peptide transmitters. However, the results obtained to date from nociceptive threshold measurements of capsaicin-treated neonatal rats have been widely varying, contradictory, and even paradoxical. Using equivalent doses of capsaicin, several groups have found small increases in thermal nociceptive thresholds (Holzer et al., 1979; Jancsó and Jancsó-Gábor, 1980; Nagy et al., 1980). Others have found increases in mechanical and chemical nociceptive thresholds (Faulkner and Growcott, 1980; Hayes et al., 1980), whereas no effect was seen on thermal nociceptive thresholds (Hayes et al., 1980). Cervero and McRitchie (1981) reported that neonatal capsaicin treatment decreases responsiveness in the paw pressure test but has no effect in the hot plate test. In the same study, quite the opposite was found in the ability of noxious stimuli to induce reflex changes in blood pressure and gastric motility; capsaicin treatment abolished these reflexes induced by noxious thermal but not mechanical stimuli applied to the abdominal skin area. All of these findings indicate that animals treated neo- 
natally with capsaicin have impairments in responses to various sorts of noxious stimuli. The reason for the disparity in the results, however, remains unclear. In previous studies the effect of usually one particular dose of capsaicin was examined and no single study has included an analysis of the effects that neonatal capsaicin administration has on all three types of noxious stimuli. The objective of the present study was to minimize the differences in methodological and treatment procedures that may be partly responsible for some of the contradictory findings. Thus, we have examined the nociceptive thermal, mechanical, and chemical thresholds of rats treated neonatally with a wide range of capsaicin doses. In addition, an attempt was made to establish a quantitative basis for changes in nociceptive thresholds by relating these changes to the extent of capsaicin-induced loss of primary afferents. In a previous paper (Nagy et al., 1983) the effects of neonatal administration of capsaicin on the fiber types and peptides contained in primary afferent systems of the rat were reported. Those results may be viewed in conjunction with the findings of the present report where the same or similarly treated animals were taken for examination of nociceptive thresholds.

\section{Materials and Methods}

The procedures for the administration of capsaicin to neonatal rats, the doses of capsaicin administered, and the various groups of animals utilized in this study are described in the previous paper (Nagy et al., 1983). Three groups of animals were taken for nociceptive threshold measurements 2 to 4 months after neonatal capsaicin treatment. Animals in group 1 were subject to measurements of nociceptive thermal thresholds only. All animals in groups 2 and 3 were taken for measurements of nociceptive thermal, mechanical, and chemical thresholds, and each animal in each group was subject to four types of tests. Nociceptive thermal thresholds were measured by the tail flick and hot plate methods. The tail flick test was conducted as previously described (Nakamura et al., 1973; Nagy et al., 1980) and involved the exposure of a limited portion of the tail to radiant heat; a ceiling of 20 sec was imposed. The hot plate test was conducted by a modification of previously described procedures (O'Callaghan and Holtzman, 1975). Animals were placed into an observation chamber consisting of clear Plexiglas walls and a metal floor which was maintained at a temperature of $54.5 \pm 0.1^{\circ} \mathrm{C}$. The latency to lick either hind paw was taken as the measure of nociceptive threshold. A ceiling of $45 \mathrm{sec}$ was imposed and only one measure was made per animal. Animals that had total analgesia and, therefore, reached this ceiling exhibited only slight swelling of the hind paw 1 day after the test. Within another few days the swelling had disappeared and no overt tissue damage was observed. A measure of the latency to lick the hind paws rather than the forepaws was used in the present study. Animals have a tendency to rear on their hind paws, thereby putting these and not the forepaws into constant contact with the heated surface, a factor which obviously contributes to the measure of latency. In the hot plate test all animals, controls or capsaicin treated, tend to lick their forepaws first. However, this reaction was never as vigorous after capsaicin treatment as in controls. Inasmuch as this was a matter of degree, it is difficult to quantitate. Mechanical nociceptive thresholds were determined as previously described (Swingle et al., 1971) using an analgesimeter (Ugo Basile, Milan, Italy). A constantly increasing pressure was applied in turn to each of the hind paws, and the nociceptive threshold was taken as the pressure required to elicit paw withdrawal or struggling behavior. This measure was taken as the average of the two hind paws and was expressed in grams. Nociceptive chemical thresholds were determined according to a slight modification of the method described by Dubuisson and Dennis (1977). Animals were taken from their home cage and given an injection of $50 \mu$ of sterile $5 \%$ formalin subcutaneously into the dorsal left forepaw. This injection procedure and the formalin itself seemed to produce minimal discomfort in animals. Rats receiving formalin injections reacted no differently than those receiving saline. After the injections, animals were housed individually for a period of $5 \mathrm{~min}$, placed in a clear Plexiglas chamber for an additional $5 \mathrm{~min}$ for the purpose of acclimatization, and finally scored for a period of $5 \mathrm{~min}$ according to the procedure described by Dubuisson and Dennis (1977). During this observation period, formalininjected animals again exhibited minimal discomfort and engaged in normal exploratory and grooming behavior. During this behavior, however, they favored the formalin-injected paw, and the degree to which this paw was favored was quantitated as previously described (Dubuisson and Dennis, 1977). Advanced healing of the small lesion produced at the site of the formalin injection was apparent 3 days after the test. The formalin test has a maximum signal to noise ratio of about 10 . That is, the nociceptive ratings obtained among control animals injected with formalin were about 10 times that obtained among control animals injected with saline; nociceptive ratings decrease with an elevation in nociceptive threshold. The above tests were conducted in the following order: hot plate, tail flick, mechanical, and chemical. A minimum time of 1 week was allowed between each of the tests. To control for the effects produced by repeated testing of animals, thresholds of capsaicin-treated rats were compared to those of vehicle or untreated rats which were taken concomitantly through all of the tests over the 4-week period.

For the analysis of the results the raw data were transformed, either by logs or reciprocals, to meet the homogeneity of variance assumption for an analysis of variance. This criterion could not be met for the nociceptive chemical threshold results where statistical analysis was conducted by the Mann-Whitney $U$ test. For all other data, analyses of variance were performed across doses for the transformed data from each nociceptive test for each of the two experimental runs (higher and lower dose ranges). Multiple comparison tests by the least significant difference procedure were carried out where significant main effects of dose were seen in the analysis of variance. The accepted level of significance was $p<0.05$ unless otherwise stated. 


\section{Results}

The results of the nociceptive threshold determinations for the set of animals designated group 3 are shown in Table I. The analysis of variance of these data revealed a significant main effect of dose in the tail flick test $(F$ $=8.9, d f=(4,65), p<0.001)$, in the hot plate test $(F=$ $8.1, d f=(4,68), p<0.001)$, and in the mechanical test $(F$ $=13.1, d f=(4,65), p<0.001)$. Significant effects for the multiple comparison in the thermal and mechanical tests, using the least significant difference procedure, and in the chemical test, using the Mann-Whitney $U$ test, are indicated in Table I. From the point of view of results obtained in previous studies, the most important observation here is that significant elevations in nociceptive thermal, mechanical, and chemical thresholds occurred at virtually all of the capsaicin doses indicated. In this group of animals significant elevations in tail flick thresholds were not seen until a capsaicin dose of $36 \mathrm{mg} / \mathrm{kg}$ was reached, and with higher doses these thresholds remained fairly constant. In most instances of the other tests, however, higher doses of capsaicin led to slightly higher nociceptive thresholds. It is of little value to compare absolute percentage changes between the various tests due to the different nature and range of the tests, the imposition of arbitrary ceilings, and the difficulty in choosing an absolute base line since no pretreatment measure is available for the capsaicin-treated animals. To present a clearer picture of these results and to emphasize an important point of the effect of capsaicin treatment on nociceptive thresholds, the scores of the individual animals in this group are shown in Figure 1. Although it is generally evident that with increasing capsaicin dose a greater number of animals exhibited higher thresholds, it is also apparent that this trend is not uniform, and this is best exemplified by the results of the hot plate test. Here, capsaicin treatment at particular doses can be seen to produce total analgesia in many animals, whereas at the same doses, some animals exhibit perfectly normal thresholds. Although less striking, this is also true of the results of the other threshold tests.

The results for animals in group 2, the low capsaicin dose series, are shown in Table II. In this group of animals the analysis of variance revealed a significant main effect of dose in the tail flick test $(F=14.6, d f=$ $(6,96), p<0.001)$, in the hot plate test $(F=4.6, d f=$ $(6,95), p<0.001)$, and in the mechanical test $(F=7.9, d f$ $=(6,96), p<0.001)$. The significant effects obtained in the multiple comparison tests using the least significant difference method for the thermal and mechanical tests and the Mann-Whitney $U$ test for the chemical test are indicated in Table II. In all of the tests the nociceptive thresholds of vehicle-treated animals were not significantly different from those of untreated controls. Small but significant elevations in nociceptive mechanical thresholds occurred at the lowest dose of capsaicin examined $(5 \mathrm{mg} / \mathrm{kg})$, whereas at the same dose thresholds in the other tests were unaltered. At doses of $10 \mathrm{mg} / \mathrm{kg}$ and higher, nociceptive chemical thresholds were significantly elevated. The comparison of both hot plate and pressure data at $15 \mathrm{mg} / \mathrm{kg}$ with those at $10 \mathrm{mg} / \mathrm{kg} \mathrm{dem}-$ onstrates significantly decreased analgesia at the higher as compared to the lower doses of capsaicin. Similar to the results in Table $I$, the doses of capsaicin required to significantly increase latencies in the tail flick test were again higher than those needed to increase thresholds in the other tests. However, for reasons we cannot explain, significant effects on tail flick latency were seen at lower doses of capsaicin in this group of animals (Table II) than in the previous group (Table I). Relevant here are the results (data not shown) from another series of animals (designated group 1) which received capsaicin doses of $12.5,25,50,75$, and $100 \mathrm{mg} / \mathrm{kg}$ and which were subject to tail flick and hot plate tests only. For animals in this group, receiving capsaicin doses of $25 \mathrm{mg} / \mathrm{kg}$ and higher, virtually the same results were obtained as shown in Table I. At a dose of $12.5 \mathrm{mg} / \mathrm{kg}$ the results were comparable to those seen at $15 \mathrm{mg} / \mathrm{kg}$ in Table II. Thus, the

TABLE I

The nociceptive thermal, mechanical, and chemical thresholds of adult rats injected neonatally with high doses of capsaicin

The results are from the animals designated group 3. All values are the mean \pm SEM of the number of animals shown in parentheses. For the thermal and mechanical threshold tests the values are the response latencies given in the units indicated. For the chemical threshold test the values are expressed in arbitrary units and were calculated as described under "Materials and Methods." Statistical analysis of all data was conducted as described in the text.

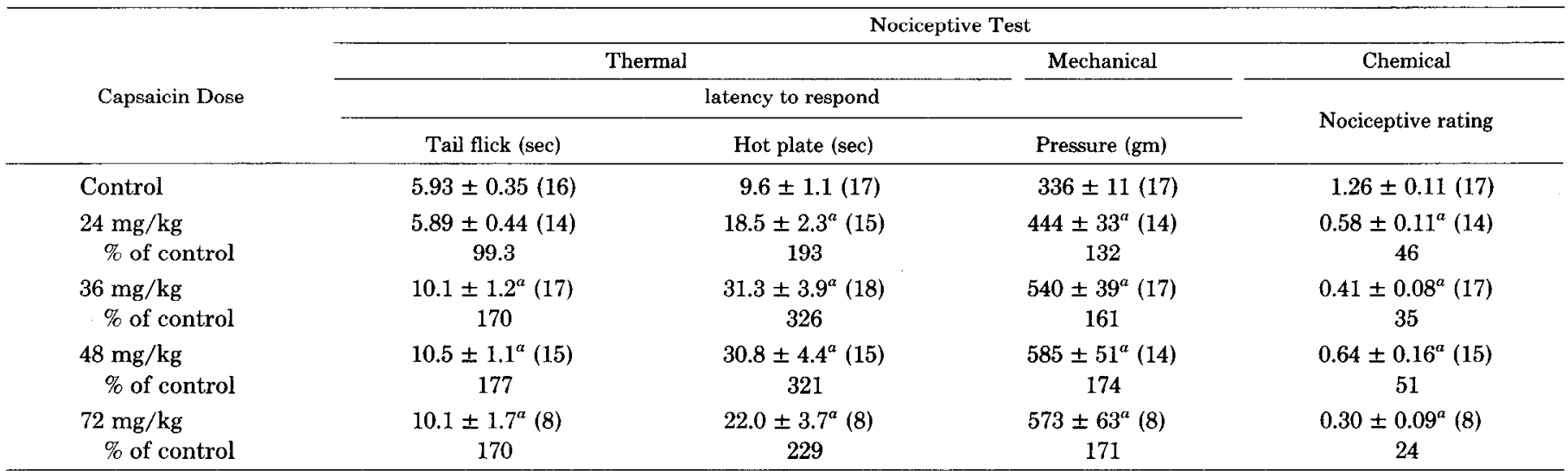

${ }^{a} p<0.05$. 

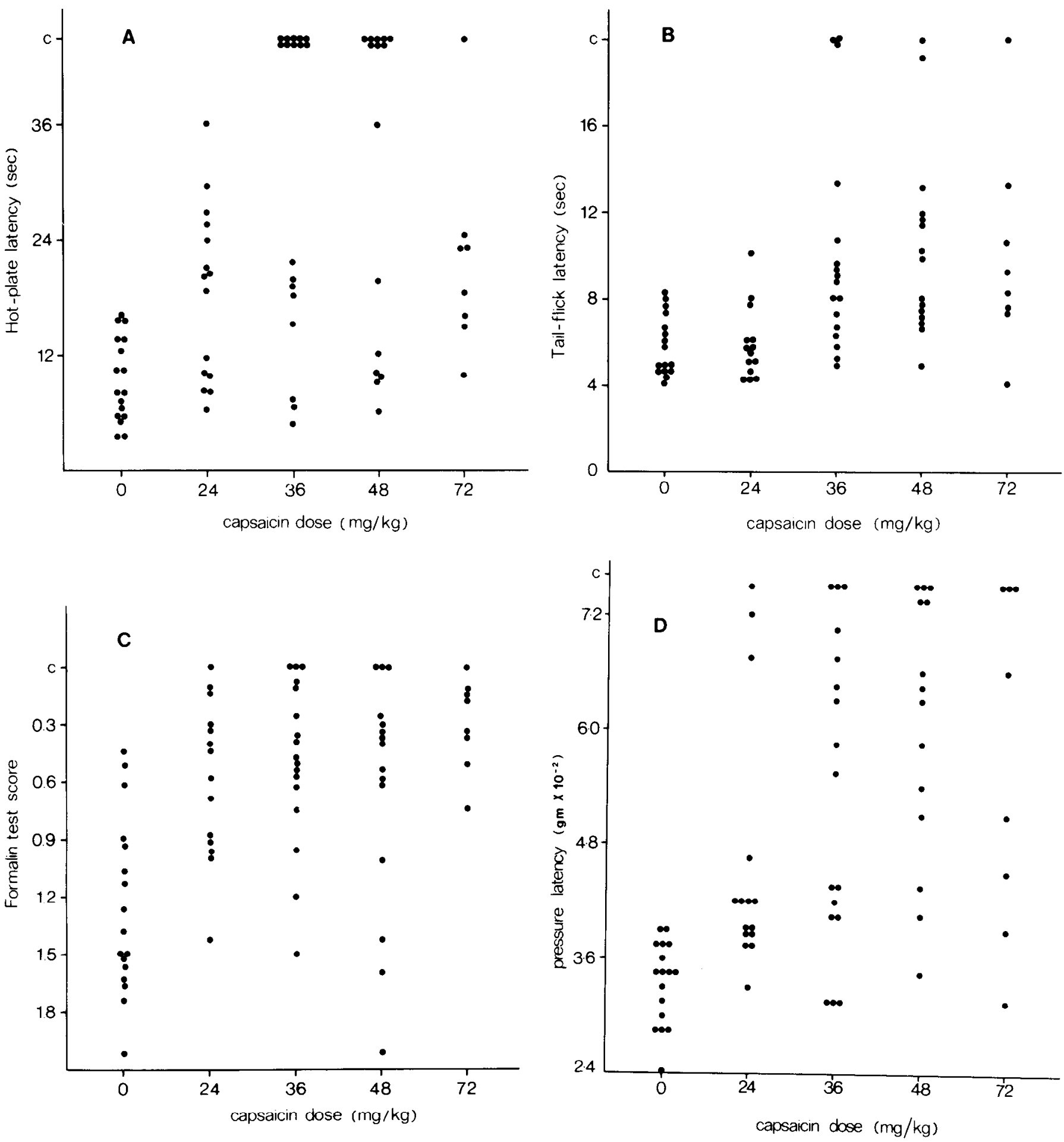

Figure 1. The nociceptive threshold scores of individual adult animals after neonatal treatment with various doses of capsaicin. These results are shown cumulatively in Table I and are taken from animals in group 3 . Each point represents a single animal. $A$, Hot plate thresholds; $B$, tail flick thresholds; $C$, chemical nociceptive rating; $D$, mechanical nociceptive thresholds. The " $C$ " on the ordinate of graphs in $A, B$, and $D$ indicates the imposed ceilings in these tests and in $C$ indicates animals with zero ratings in the formalin test. The ascending ordinate in the formalin test $(C)$ shows decreasing nociceptive rating and thus increasing analgesia to facilitate visual comparison with the results of the other tests.

discrepant tail flick test results in Tables 1 and 2 may simply indicate that doses of capsaicin between 20 and $30 \mathrm{mg} / \mathrm{kg}$ are the threshold for causing significant effects in this test.

It was of interest to determine whether at a particular dose of capsaicin, or among the controls, the behavior of individual animals in one test correlated with that of any other test. Analysis of the combined data from groups 2 and 3 using Spearman's rank correlation method at each dose level revealed only 3 of 72 possible significant correlations between nociceptive threshold tests at the various doses. This analysis suggests that the four different 
TABLE II

The nociceptive thermal, mechanical, and chemical thresholds of adult rats injected neonatally with low doses of capsaicin

The results are from the animals designated group 2. Values indicate the means \pm SEM of the number of animals per group shown in parentheses. All other details are as described in Table 1 .

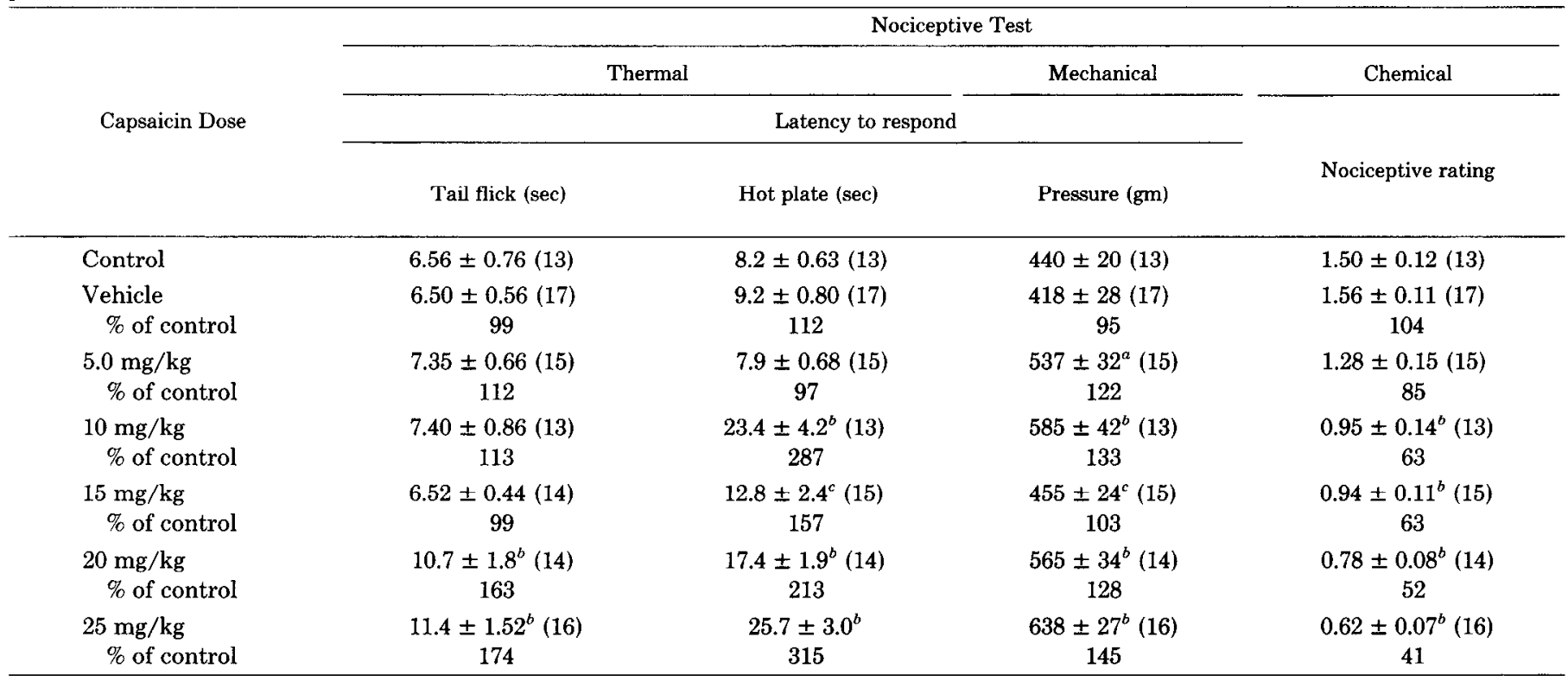

${ }^{a} p<0.05$, compared to uninjected controls.

${ }^{b} p<0.01$, compared to uninjected controls.

${ }^{c} p<0.01$, compared to threshold at $10 \mathrm{mg} / \mathrm{kg}$.

nociceptive tests measure quite different behavioral responses to noxious stimuli. Because the behavior of an animal on any one test could not be predicted from its behavior on any other, no attempt was made to depict by identification the behavior of each animal in each of the tests shown in Figure 1.

\section{Discussion}

One of the major findings of the present study is that capsaicin treatment of neonatal rats causes analgesia to all three types of nociceptive stimuli examined: thermal, mechanical, and chemical. Our results concerning the antinociceptive effects of capsaicin to noxious chemical and mechanical stimuli are in agreement with those of others (Faulkner and Growcott, 1980; Hayes et al., 1980; Cervero and McRitchie, 1981), and we further showed that similar effects can be achieved with much lower doses of capsaicin than previously used. However, there are inconsistencies regarding the effects of neonatal capsaicin treatment on nociceptive thermal thresholds. At large doses of capsaicin either no effects (Hayes et al., 1980; Cervero and McRitchie, 1981) or only small increases in thermal thresholds have been reported (Holzer et al., 1979; Nagy et al., 1980; Jancsó and Jancsó-Gábor, 1980), whereas here, profound thermal analgesia was found among animals given similar or smaller capsaicin doses. Although it is possible that the source of these inconsistencies resides in methodological differences either in treatment of animals with capsaicin or in nociceptive threshold testing, the results of the present study have partly ruled these out and, at the same time, provided another possibility. We have shown that increasing the dose of capsaicin given to neonatal animals increases the degree of analgesia the animals exhibit when they are analyzed in groups. However, at any particular dose of capsaicin, animals within a group were found to have a wide range of thresholds to noxious stimuli. It is unlikely that this variability in thresholds was produced by a differential neurotoxic action of capsaicin in animals receiving similar doses of this agent inasmuch as reproducible and consistent depletions of primary afferent fibers and their constituent peptides were observed in the dorsal roots of the present set of animals (Nagy et al., 1983). Thus, it is possible that variability in nociceptive thresholds is a fundamental and inherent principle of the elimination of certain primary afferent fibers so early in development. This may i.،dicate the complex nature of the changes that may occur in primary afferent connectivity or in the CNS after neonatal capsaicin treatment.

The results of the present study, together with those in the previous paper (Nagy et al., 1983), allow some conclusions to be drawn regarding the degree of primary afferent fiber loss that must occur to precipitate the events leading to altered nociceptive thresholds. Since a negligible loss of myelinated fibers was found in the dorsal roots of animals receiving a capsaicin dose of 25 $\mathrm{mg} / \mathrm{kg}$, we conclude that in animals receiving the lower doses of 20 and $10 \mathrm{mg} / \mathrm{kg}$ the depletion of only unmyelinated primary afferents occurred. From the observation that nociceptive thermal, mechanical, and chemical thresholds were elevated in animals given these lower doses of capsaicin, it can be concluded that the depletion of unmyelinated fibers alone is sufficient to cause changes in nociceptive thresholds. Insofar as the effects of higher doses of capsaicin are conrerned, it is not possible to decide whether the profound analgesia seen in some animals receiving these doses is due to a greater loss of 
unmyelinated fibers, to the loss of myelinated fibers, or to the loss of both of these from dorsal roots. In general, when considering average total fiber depletions and average nociceptive thresholds of a large number of animals receiving particular doses of capsaicin, larger doses did produce greater total fiber loss and higher nociceptive thresholds. However, in some animals of group 1 for which both thermal threshold results and fiber counts in dorsal roots were available, in individual animals no correlation could be found between reaction latency and the degree of either unmyelinated or myelinated fiber depletions.

The merit of deductions of neurotransmitter function in primary afferents based on behavioral, anatomical, and neurochemical observations has been discussed previously (Nagy, 1982). The present observation of significantly elevated nociceptive mechanical thresholds at a capsaicin dose of $5 \mathrm{mg} / \mathrm{kg}$, taken together with the findings in the previous paper (Nagy et al., 1983) of significant depletions of unmyelinated fibers and the absence of reductions of substance $P$ and somatostatin levels in dorsal roots at this dose, may suggest that these peptides do not play a role in the sequence of events leading to the behavioral manifestation of mechanical nociception. From the present set of results it is difficult to draw further conclusions regarding the function of these peptides in primary afferents.

All of the above conclusions must be qualified by the fact that only the fiber depletions in the 3rd lumbar dorsal root were analyzed, that all of the lumbar and sacral roots were taken for peptide determinations, and that nociceptive threshold measurements reflect activity in many facets of the nervous system. Further work is required to determine whether the generalizations we have made are justified.

\section{References}

Bessou, P., and E. R. Perl (1969) Response of cutaneous sensory units with unmyelinated fibers to noxious stimuli. J. Neurophysiol. 32: 1025-1043.

Burgess, P. R., and E. R. Perl (1973) Cutaneous mechanoreceptors and nociceptors. In Handbook of Sensory Physiology. Vol. 2: Somatosensory System, A. Iggo, ed., pp. 31-78, Springer-Verlag, Berlin.

Cervero, F., and H. A. McRitchie (1981) Neonatal capsaicin and thermal nociception: A paradox. Brain Res. 215: 414-418.
Dubuisson, D., and S. G. Dennis (1977) The formalin test: A quantiative study of the analgesic effect of morphine, meperidine and brain stem stimulation in rats and cats. Pain 4: 161-174.

Faulkner, D. C., and J. W. Growcott (1980) Effects of neonatal capsaicin administration on the nociceptive response of the rat to mechanical and chemical stimuli. J. Pharm. Pharmacol. 32: 656-657.

Hallin, R. G., and H. E. Torebjork (1973) Electrically induced $\mathrm{A}$ and $\mathrm{C}$ fibre responses in intact human skin nerves. Exp. Brain Res. 16: 309-320.

Hayes, H. G., J. W. Scadding, M. Skingle, and M. B. Tyers (1980) Effect of neonatally administered capsaicin on nociceptive thresholds and dorsal horn substance $P$ levels in conscious rats and mice. J. Physiol. (Lond.) 305: 99.

Henry, J. L. (1976) Effects of substance $P$ on functionally identified units in cat spinal cord. Brain Res. 114: 439-451.

Holzer, P., I. .Jurna, R. Gamse, and F. Tembeck (1979) Nociceptive threshold after neonatal capsaicin treatment. Eur. J. Pharmacol. 58: 511-514.

Jancsó, G., and A. Jancsó-Gábor (1980) Effect of capsaicin on morphine analgesia-Possible involvement of hypothalamic structures. Naunyn Schmiedebergs Arch. Pharmacol. 311: 285-288.

Jancsó, G., E. Kiraly, and A. Jancsó-Gábor (1977) Pharmacologically induced selective degeneration of chemosensilive primary sensory neurons. Nature 270: 741-743.

Nagy, J. I. (1983) Capsaicin: A chemical probe for sensory neuron mechanisms. In Handbook of Psychopharmacology, L. L. Iversen, S. D. Iversen, and S. H. Snyder, eds., pp. 185235, Plenum Press, New York.

Nagy, J. I., S. R. Vincent, W. A. Staines, H. C. Fibiger, T. D. Reisine, and H. I. Yamamura (1980) Neurotoxic action of capsaicin on spinal substance P neurons. Brain Res. 186: 435444.

Nagy, J. I., L. L. Iversen, M. Goedert, D. Chapman, and S. P. Hunt (1983) Dose-dependent effects of capsaicin on primary sensory neurons in the neonatal rat. J. Neurosci. 3: 399-406.

Nakamura, K., R. Kuntzman, A. C. Maggio, V. Augulis, and A. H. Conney (1973) Influence of 6-hydroxydopamine on the effect of morphine on the tail-flick latency. Psychopharmacologia (Berlin) 31: 177-189.

O'Callaghan, J. P., and S. G. Holtzman (1975) Quantification of analgesic activity of narcotic antagonists by a modified hot plate procedure. J. Pharmacol. Exp. Ther. 192: 497-505.

Swingle, K. F., T. J. Grant, and D. C. Kuam (1971) Quantal responses in the Randall-Selitto assay. Proc. Soc. Exp. Biol. Med. 137: 536-538.

Torebjork, H. E., and R. G. Hallin (1974) Identification of afferent $\mathrm{C}$ units in intact human skin nerves. Brain Res. 67: 387-403. 